\title{
Efficiency of Group Mindfulness-based Stress Reduction Program on the Guality of Life and Job Stress Among Emergency Medical Technicians
}

Mojgan Maghareh Abed, Master of General Psychology, Islamic Azad University, Khorasan Branch, Isfahan, Iran.

ㄴ) Maryam Azadi, ( ${ }^{*}$ Corresponding author), Master of General Psychology, Faculty of Education and Psychology, Islamic Azad University, Arak Branch, Arak, Iran. M.azadi_61@yahoo.com

Maryam Dehghan, Assistant Professor, Department of Internal Medicine, Shahid Sadoughi University of Medical Sciences, Yazd, Iran.

\section{Abstract}

Background and aims: Emergency medical technicians experience more job stress due to being exposed to various emergency conditions. The present study was conducted to the efficiency determination of the group mindfulness-based stress reduction program, on the quality of life and job stress among emergency medical technicians.

Methods: The method used for the research was quasi-experimental. The statistical population were all, emergency medical technicians in Isfahan in 2019, of whom 30 were selected using convenience sampling, and given the inclusion criteria, they were divided into case $(n=15)$ and control groups $(\mathrm{n}=15)$. The case group, received mindfulness-based stress reduction training, and the control group, received no interventions. MBSR therapy was conducted in 8 sessions of 120 minutes once a week. The research instruments were, the Quality of Life Scale developed by Ware, Kosinski and Keller (1996) and the Job Stress Scale developed by Kahn, Wolf, Quinn, Snook and Rosenthal (1964). Data were analyzed in SPSS 23.

Results: The results of ANOVA showed that the mindfulness-based stress reduction, caused an increase in the mean score of the quality of life among emergency medical technicians in the case group compared to the control $(\mathrm{P}<0.05, \mathrm{~F}=55.77)$. In addition, results of ANCOVA indicated that, mindfulnessbased stress reduction, reduced the mean score of job stress in the emergency medical technicians $(\mathrm{P}<0.05, \mathrm{~F}=12.70)$.

Conclusion: Given the results of the study, teaching the mindfulness-based stress reduction skills by encouraging people to practice them frequently, and focusing on body and mind, can help the emergency medical technicians, to get rid of preoccupation with threatening thoughts and worries about the job performance, and increase their quality of life.

Conflicts of interest: None

Funding: None

\section{Keywords}

Mindfulness-based Stress

Reduction

Quality of Life

Job Stress

Emergency Medical Techni-

cians

Received: 2020/02/17

Accepted : 2020/12/12 
Background and Aims

One of the stressful stimuli for human beings, is their job and working conditions. There is more stress in jobs with more human relations. In psychology, stress is defined as 'being under pressure. Job stress, could create physical or behavioral problems and affect the people's quality of life. Physicians are more commonly exposed to physical and mental health disorders, due to their type of work, lack of sleep, and clinical shifts. Among physicians and medical staff in various wards, the emergency medical technicians are more exposed to stressful environments where working is difficult, such as places full of injured people, seriously ill patients, and so on. Stressful physical, mental and psychological stimuli, causes the emergency medical technicians to face various dangers, such as accidents, mistakes, and injuries. Therefore, the level of stress and job dissatisfaction is higher among the emergency medical technicians compared to other occupations. Job stress endangers a person's health by causing physical, psychological and behavioral complications, and failure in paying attention to the state of occupational medical stress can lead to absenteeism, delays, reduced efforts, and increased occupational errors. The quality of life is a multidimensional and complex structure that includes mental and objective factors, and usually refers to a person's feelings, mental perception and evaluation of life in various areas, such as job status, economy and relationships with others etc. Therefore, one of the most effective preventive and effective methods to maintain health and psychological well-being of people, is mindfulness-based stress reduction therapy. MBSR therapy is a form of concentration to reduce stress. Mindfulness-based interventions are increasingly used in both mental and physical health, aiming at reducing the psychological symptoms of anxiety and increasing the quality of life. Results of the study by Linda on nurses in Spain, indicated a relation between stress and nurses' health. In his study, Cosio confirmed the effects of mindfulness-based stress management on quality of life of the patients. Studies conducted on the role of MBSR in the work environment indicate that, these interventions play a crucial role in workrelated outcomes, such as task performance, improving metacognitive beliefs, and psychological health. The present study was conducted to determining the efficiency of the group mindfulness-based stress reduction program on the quality of life and job stress among emergency medical technicians.

\section{Method}

The research method was quasi-experimental with a pretest-posttest design and case and control groups. The statistical population was all emergency medical technicians in Isfahan in 2019 of whom 30 were selected using convenience sampling and given the inclusion criteria, they were divided into case $(n=15)$ and control groups $(n=15)$. The case group received mindfulness-based stress reduction training, and the control group received no interventions. Inclusion criteria signed an informed consent to participate in the study, having no acute physical or mental illness at the discretion of the psychologist and physician. The technicians working in the road emergency department, scoring higher or lower than average in the quality of life and job stress questionnaires and did not receive any psychological and pharmacological treatments during the last months. Exclusion criteria, included absenteeism for more than 3 sessions and dissatisfaction with participation in the study. MBSR therapy was conducted in 8 sessions of 120 minutes, once a week. After the sessions, posttest was performed on case and control groups. The research instruments were, the Quality of Life Scale developed by Ware, Kosinski and Keller (1996) and the Job Stress Scale developed by Kahn, Wolf, Quinn, Snook and Rosenthal (1964). The Quality of Life Scale (Short Form $12=\mathrm{SF}-12$ ) is a 12 -item scale, which measures the quality of life of people in both physical and mental dimensions in terms of health perception, physical functions, physical health, emotional problems, physical pains, social functions, vitality and vital energy and mental health. Minimum and maximum scores in this scale are 12 and 48, where higher scores indicate better quality of life. This scale was translated into Persian by Montazeri et al. and its validity and reliability were between 0.72 and 0.83 for physical and mental performance. In the present study, reliability of the questionnaire using Cronbach's alpha was 0.87 and 0.86 for physical and mental performance, respectively. Another instrument used in the present study was, the Job Stress Scale developed by Kahn et al. This scale included 15 items of 6 points ( $1=$ Never, $6=$ Always). The study by Yusefi, Nayeb Zadeh and Islami on 30 accountants, indicated that the scale is of good content validity. Exploratory factor analysis was used to evaluate the validity of the structure. KMO was 0.88 and Bartlett's test was significant at $\mathrm{P}<0.001$. In the present study, Cronbach's alpha of the scale was 0.74 . Descriptive and inferential data analyses were conducted. The descriptive statistics, included mean and standard deviation, and the inferential statistics included analysis of covariance tests, which were used to test the research hypotheses; Kolmogorov-Smirnov and Shapiro-Wilk tests were used to evaluate the normality of the variable distribution, and the Box's $\mathrm{M}$ and Levene's tests were used to observe ANOVA assumptions. Data were analyzed in SPSS 23.

\section{Results}

To investigate the effects of the independent variable (mindfulness-based therapy) on dependent 
variables (quality of life and job stress), the multivariate analysis of covariance (MANOVA) was employed. First, items of the test were investigated. For the normality of data, the Shapiro-Wilk test was used. Given that, the significance level for the job stress and quality of life, was greater than 0.05 (job stress, $\mathrm{Z}=1.338$ and $\mathrm{a}=0.056$ and quality of life $\mathrm{Z}=0.785$ and $\mathrm{a}=0.568$ ). The data distribution is normal. Levene's test was used to evaluate the homogeneity of the variances, and all the variables were greater than 0.05 (job stress $\mathrm{F}=0.253$ and $\mathrm{a}=$ 0.618 and quality of life $\mathrm{F}=0.861$ and $\mathrm{a}=0.459$ ); therefore the assumption of equality of variances is observed. In addition, the Box's $M$ test, which was used to investigate the assumption of homogeneity of variance-covariance matrices, indicated that the hypothesis stands, and ANOVA could be used $\left(\mathrm{F}=37.828, \mathrm{M}_{\text {Box }}=6.321, \mathrm{a}=0.068\right)$. The results of ANOVA showed that the mindfulness-based stress reduction intervention, led to an increase in the mean score of the quality of life (99.10) among the emergency medical technicians in the case group compared to the control $(\mathrm{P}<0.05, \mathrm{~F}=55.77)$. Thus, 0.667 of the changes that occurred in the scores of the quality of life, were due to the mindfulness-based stress training program. In addition, the results of ANCOVA, showed that the mindfulness-based stress reduction intervention, led to a decrease in the mean score of job stress $(27.50)(\mathrm{P}<0.05, \mathrm{~F}=12.70)$ in the emergency medical technicians. 0.485 of the changes in scores of job stress, was due to this intervention.

\section{Conclusion}

Accordingly, there was a significant relationship between using the mindfulness-based stress reduction with increased quality of life, and decreased job stress among emergency medical technicians To explain the results, the mindfulness-based stress reduction is defined as a therapeutic process, which emphasizes paying active attention to the feelings and thoughts of the patient, without judging or valuing those feelings and thoughts. With attention following mindfulness, one enters a reinforcing situation that himself/herself has created. This process, ultimately leads to the adaptation and improvement of thoughts, mood and overall quality of life. In addition, extreme fatigue and shift changes in the emergency medical technicians, causes a decrease in their physical strength and as a result, reduces their physical function; on the other hand, they reduce the psychological dimensions related to the quality of life in these people. Therefore, teaching mindfulness-based stress reduction skills by encouraging people to practice them frequently, and focusing on body and mind, can help the emergency medical technicians get rid of preoccupation with threatening thoughts and worries about their job performance, and increase their quality of life. In addition, being mindful, helps people to get rid of stresses and worries; So, they are not easily affected by environmental factors and conditions, and could use their talents and creativity to express themselves, and deal with adverse conditions. Therefore, people with this characteristics, consider themselves lovable and valuable and do not procrastinate in doing things, so, they suffer from less job stress. Results of the present study showed that teaching MBSR, could be employed as an effective non-pharmacological method to reduce job stress, and improve the quality of life among emergency medical technicians. It is expected that, with the proper implementation of these interventions, the anxiety and job stress of the emergency medical technicians reduces, and their job satisfaction, quality of life and resilience in emergency situations improves. One of the limitations of the present study was the lack of a follow-up period to indicate the effects of training stability and continuity of the treatment results. Therefore, it is suggested to consider the follow-up plans in future studies. Another limitation of the present study was selfreporting, which is affected by social desirability. So, findings should be generalized with care. Therefore, it is recommended to use more valid clinical assessment methods for the future studies, such as interviews, to measure variables as well as reinforcing the results.

\section{Conflicts of interest \\ None}

Financial resources

None

\section{How to cite this article:}

Mojgan Maghareh Abed, Maryam Azadi, Maryam Dehghan. Efficiency of Group Mindfulness-based Stress Reduction Program on the Guality of Life and Job Stress Among Emergency Medical Technicians. Iran Occupational Health. 2021 (01 Apr);18:8.

\footnotetext{
*This work is published under CC BY-NC-SA 3.0 licence
} 


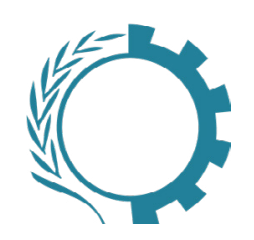

مقاله يزؤششى

http://ioh.iums.ac.ir

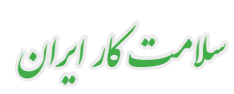

N/IK.. - 11 د د

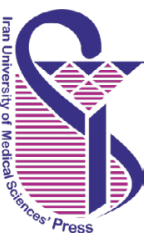

اثربخشى بر نامه كاهش استر س مبتنى بر ذهن آكاهى بله شيوه تروهى بر كيفيت زندكى و استر س

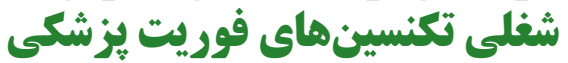

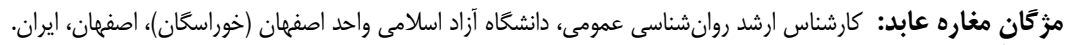

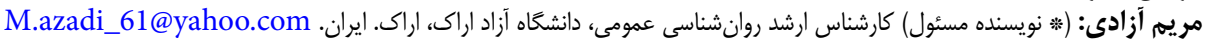

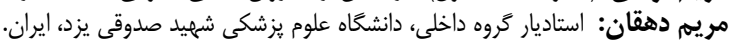

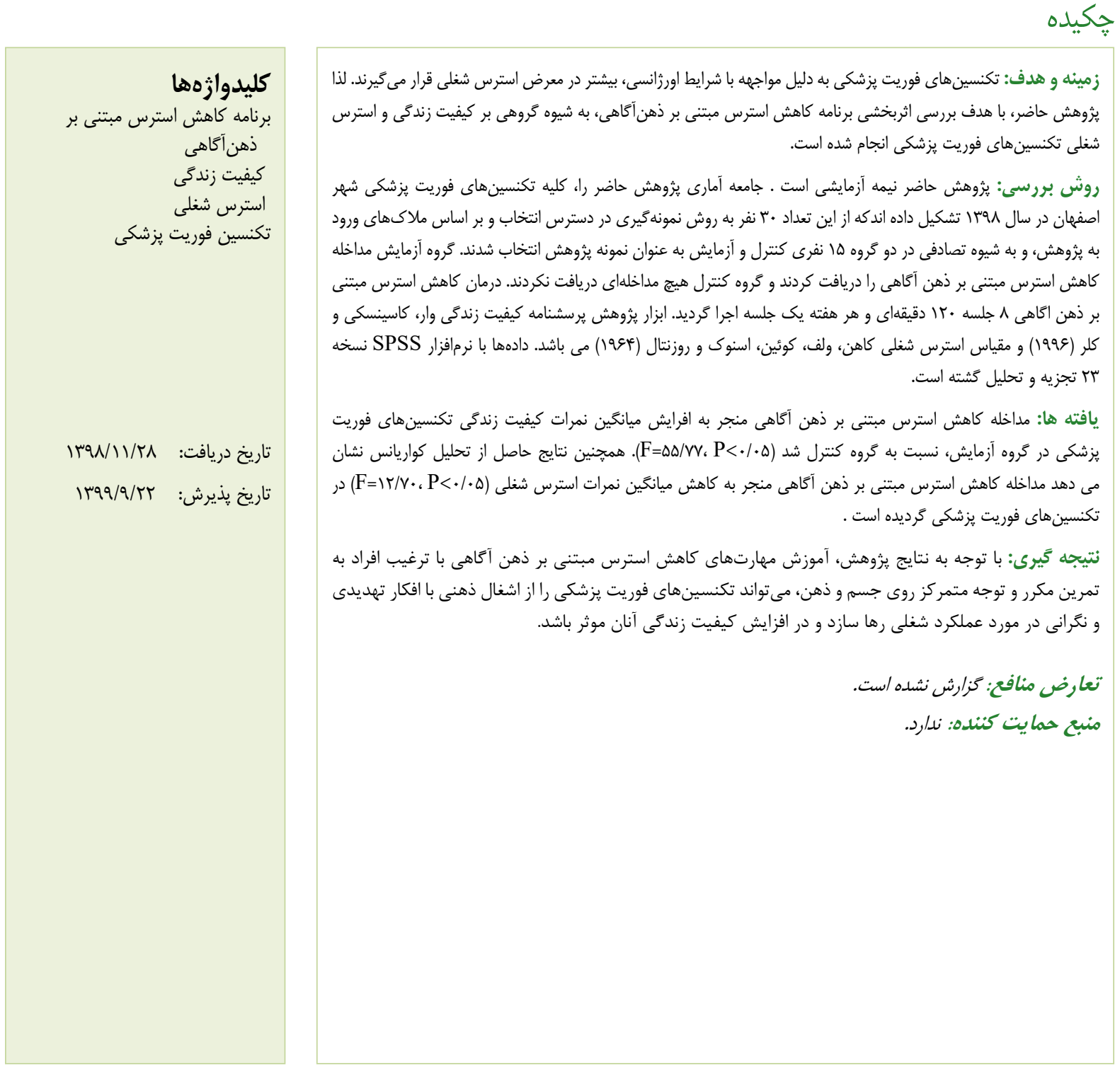

شيوه استناد به اين مقاله:

Mojgan Maghareh Abed, Maryam Azadi, Maryam Dehghan. Efficiency of Group Mindfulness-based Stress Reduction Program on the Guality of Life and Job Stress Among Emergency Medical Technicians. Iran Occupational Health. 2021 (01 Apr);18:8.

*تشار اين مقاله به صورت دسترسى آزاد مطابق با CC BY-NC-SA 3.0 صورت ترفته است 
مىباشد (9). محيط كار از محركهاى فيزيكى، روانى و

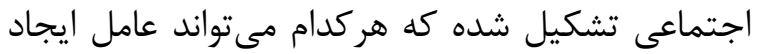

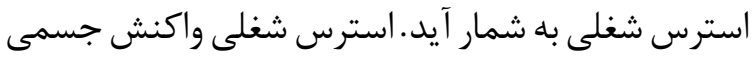

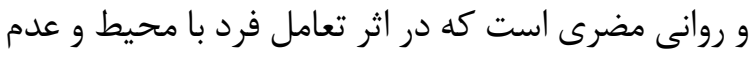

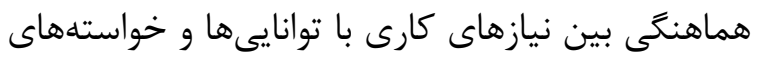

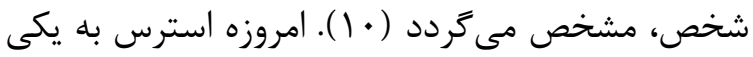

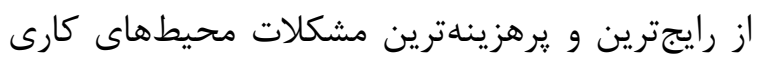

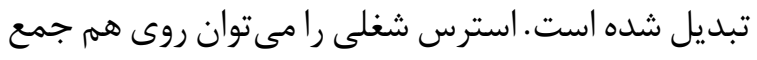

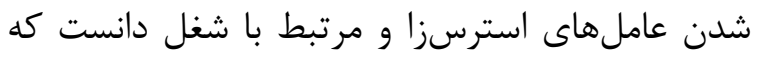

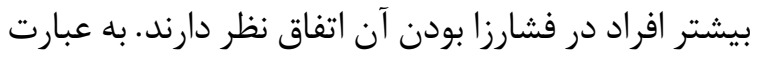

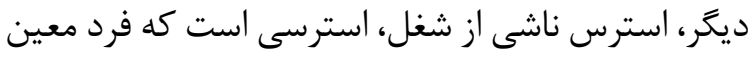

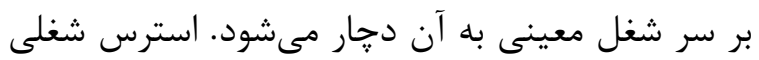

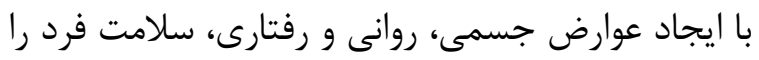

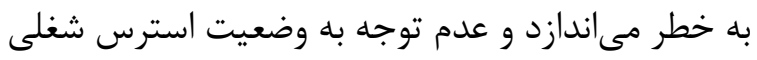

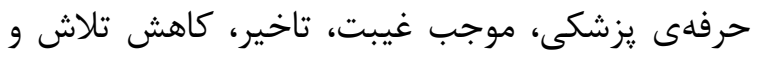

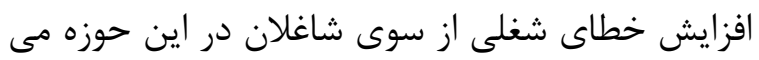

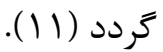
مشاغل درمانى ممكن است كيفيت زندكى بزشكان،

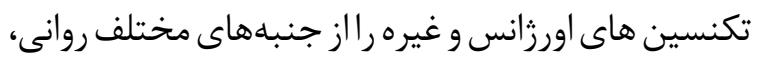

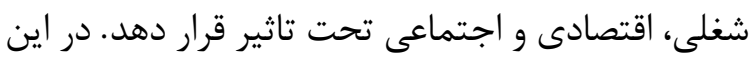

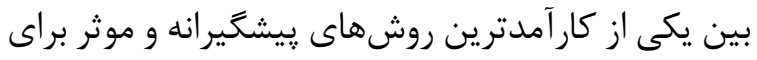

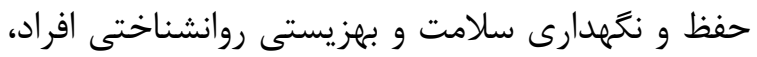

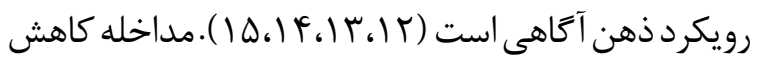

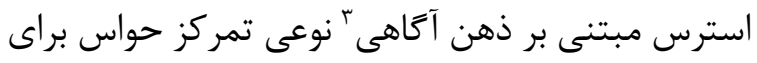

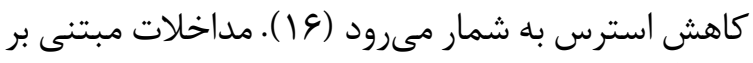

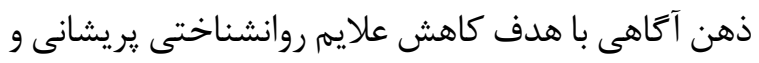

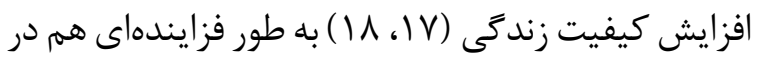

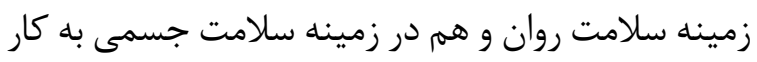

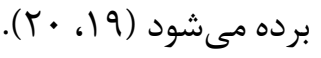

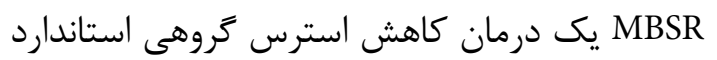

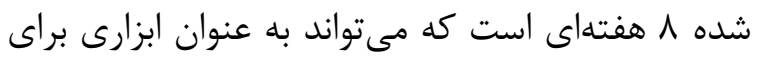

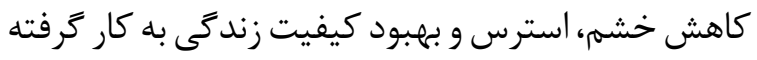

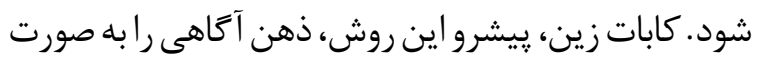

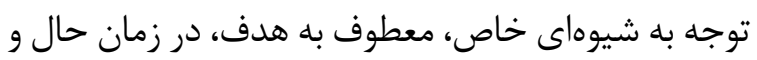

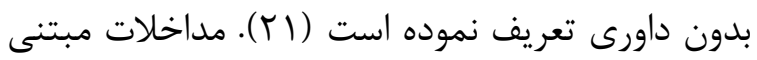

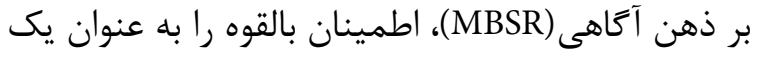

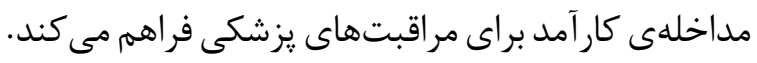

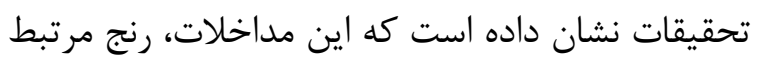

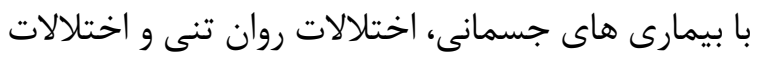

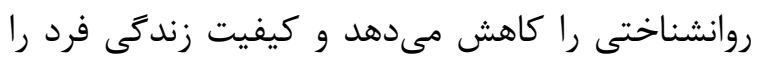

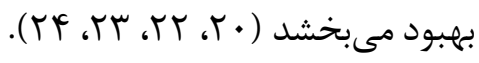

3 .Mindfulness based stress reduction (MBSR)
مقاله

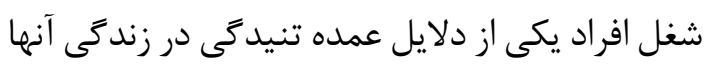

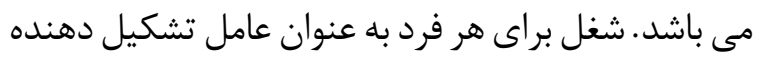

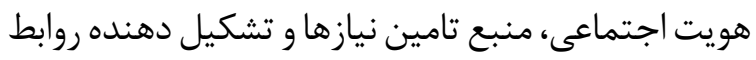

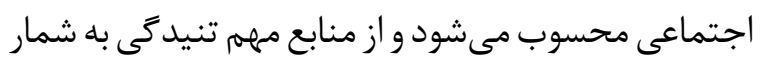

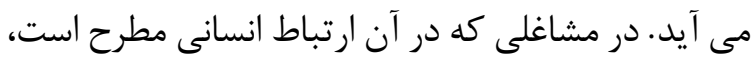

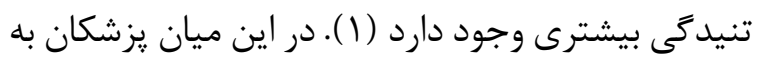

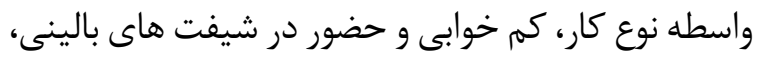

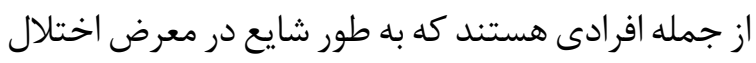

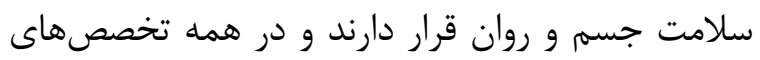

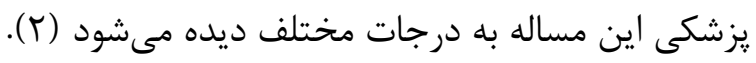

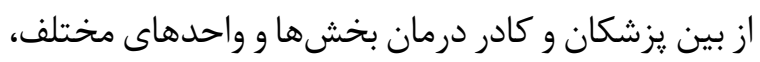

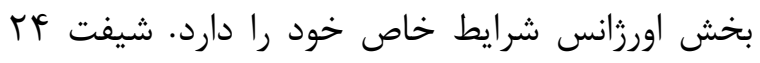

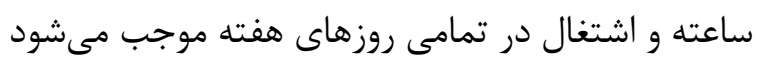

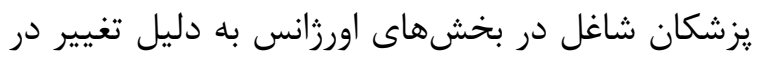

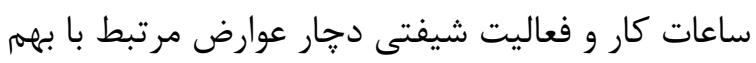

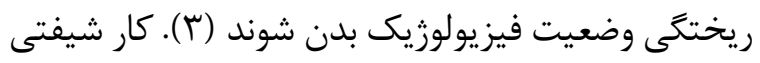

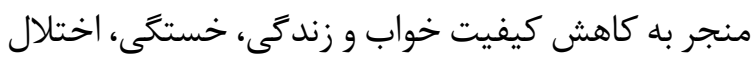

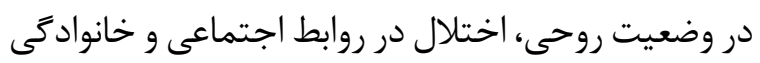

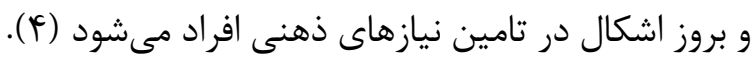

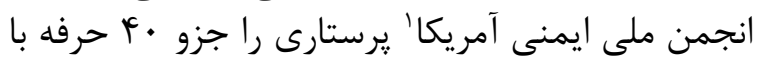

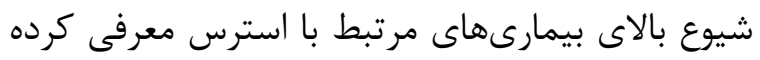

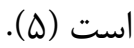

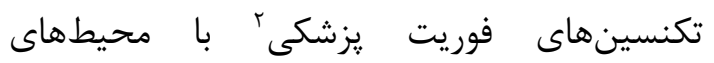

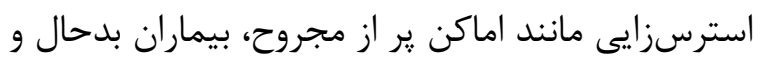

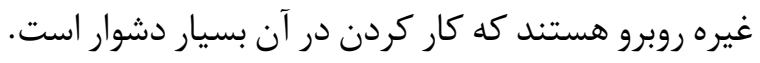

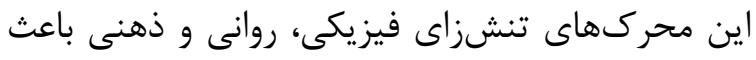

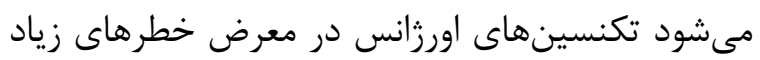

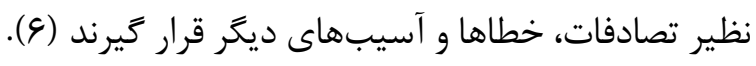

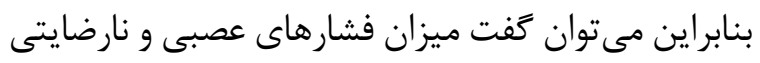

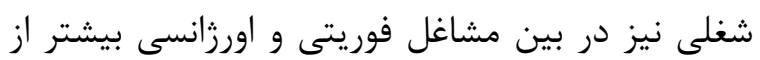

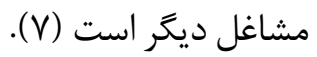

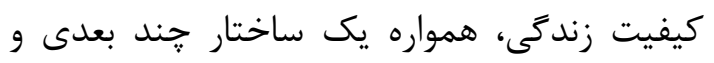

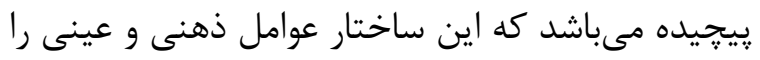

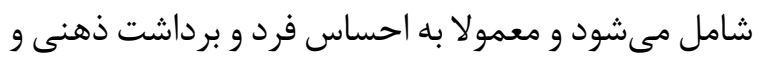

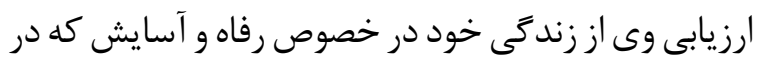

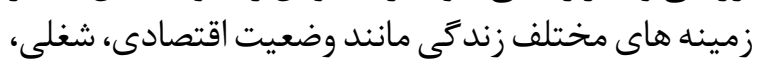

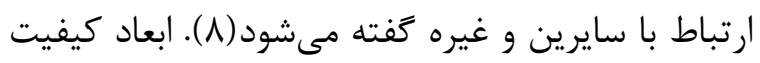

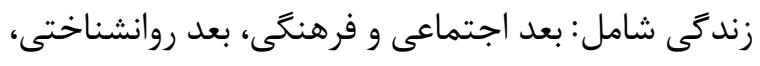

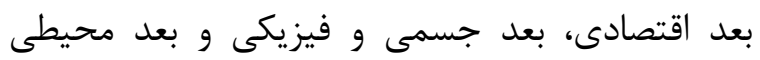

1. American National Safety Association

2. Emergency Medical Technicians

Iran Occupational Health. 2021 (01 Apr);18: 8. 
الف. طرح برروهى

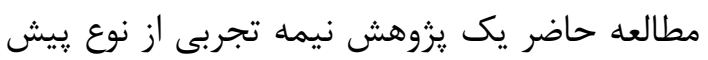

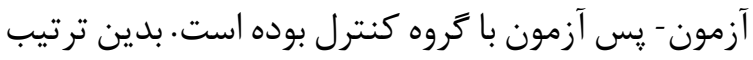

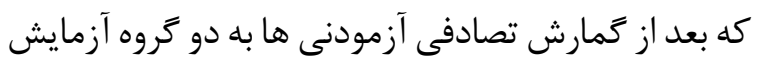

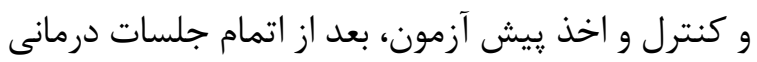

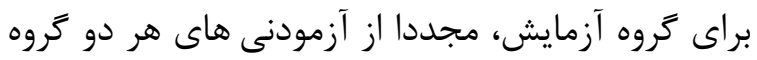
يس آزمون گَرفته شد.

\section{ب. شركت كنندكان}

جامعه آمارى اين يزوهش شامل تمامى تكنسينهاي

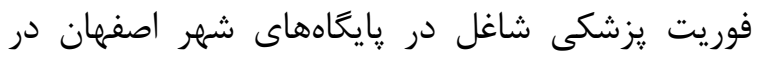

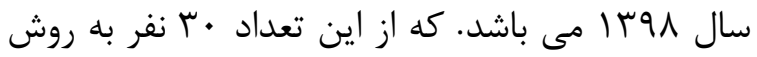

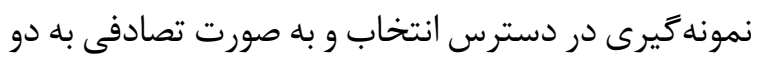

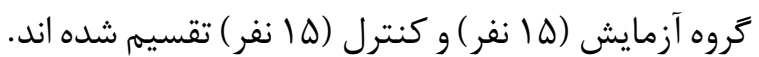

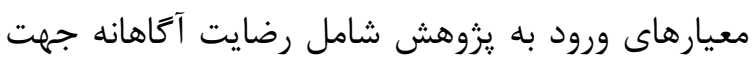

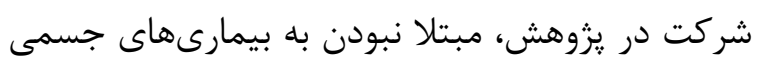

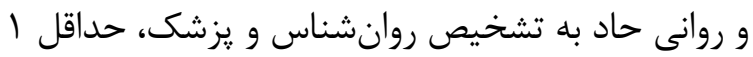

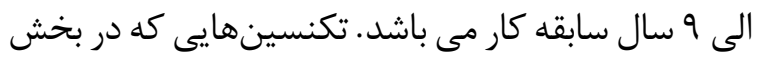

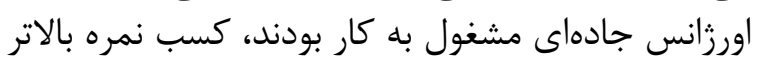

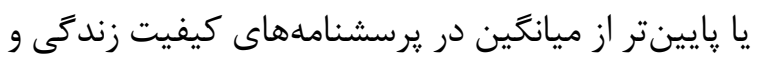

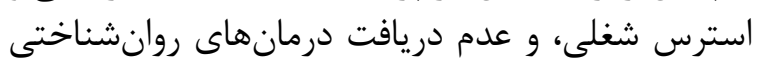

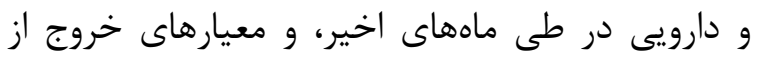

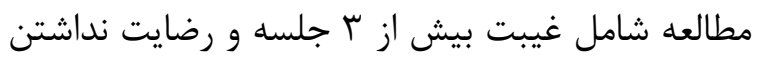

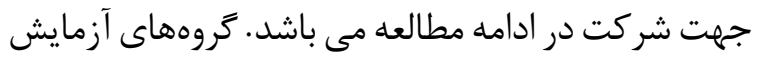

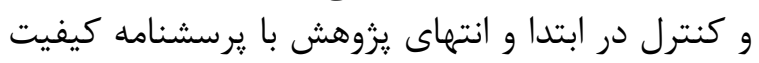
زندگى و استرس شغلى مورد ارزيابى قرار كرفتيندا.

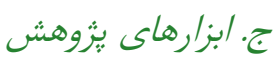

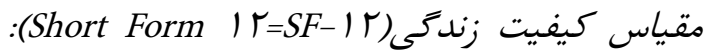

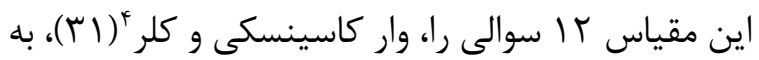

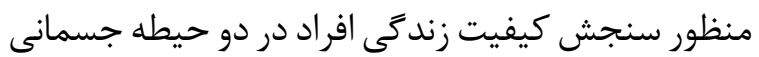

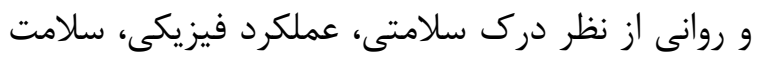

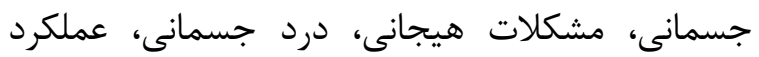

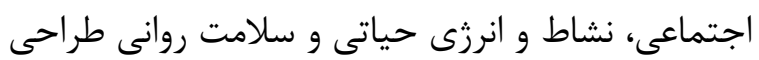

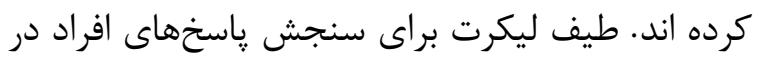

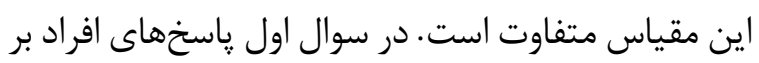

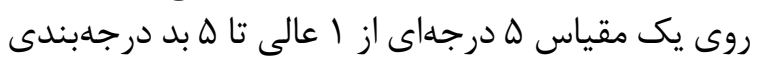

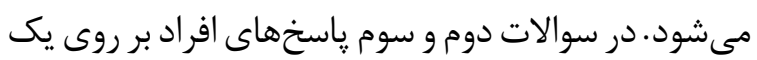

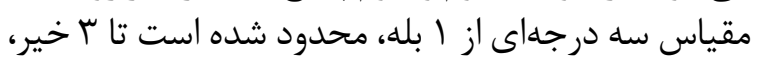

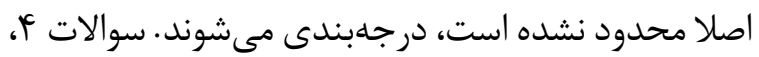

4. War, Kasinski \& Keller
يزوهشهاى متعددى رابطه بين كيفيت زندگى و و استرس شغلى را بررسى كرده اند:

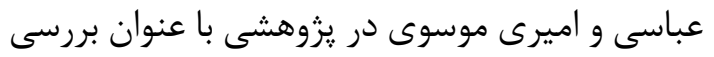
رابطه استرس شغلى با كيفيت زند

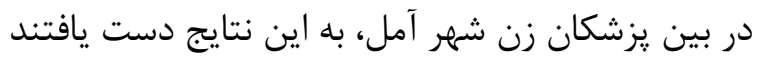

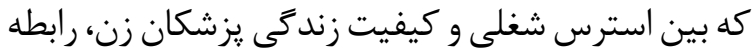

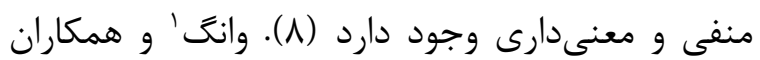
به دنبال مطالعه ى خود بر روى وضعيت سلامت ودود دارد روانى،

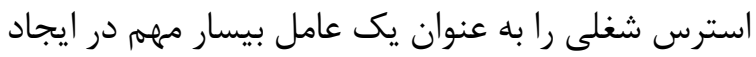

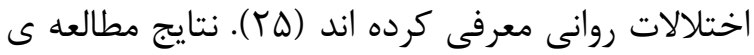

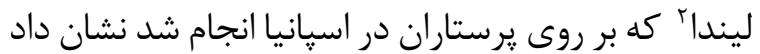

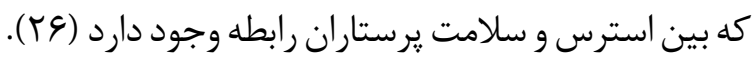
عابدى و همكاران رابطه ى بين استرس شغلى وسى و نارضايتى از كار و ارتباط آن با عوامل فردى و و حوادث ران ران ران در بين كاركران كارخانه بررسى كردند. نتايج نشان دان داد كان كه بين استرس شغلى و رضايت شغلى رابطه منفى و معنادارى

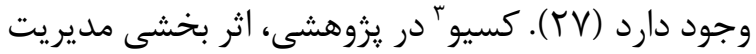

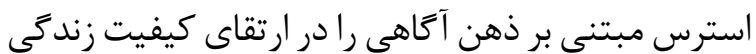
تاييد كرده است (r人).

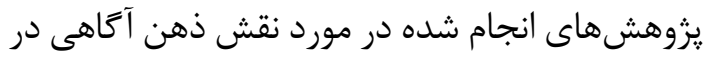

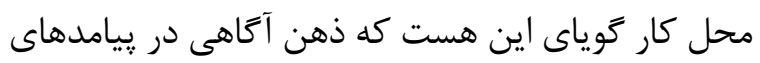
مرتبط با كار مثل اجراى وظايف، بهبود باوي هاورهاى فر اشناختى دهن

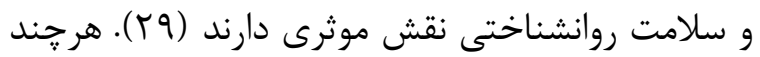

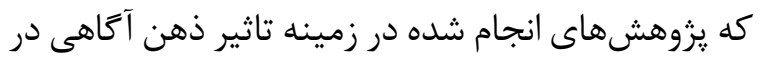
كاهش استرس شغلى و افزايش خودكارآمدى و و كيفيت

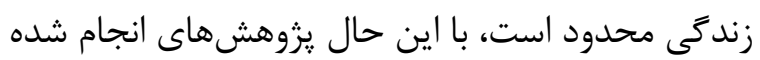

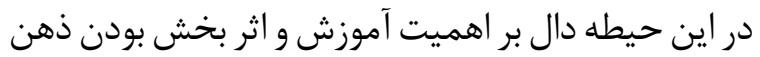

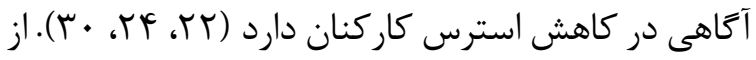

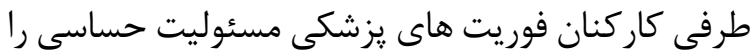

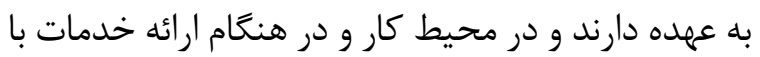

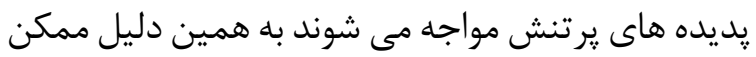

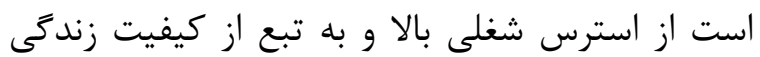

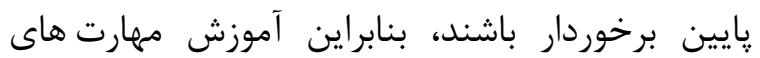

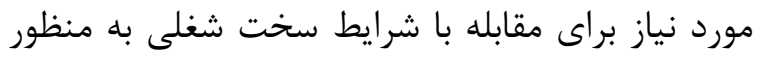

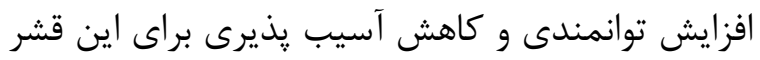

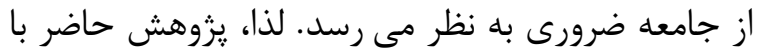

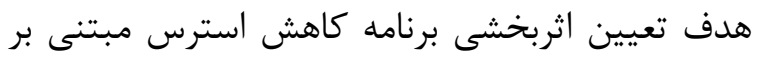

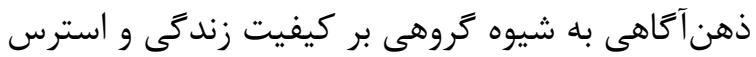
شغلى تكنسين هاى فوريت يزشكى صورت گرفته است

1. Wang
2. Linda
3. Cosio 
اثربخشى برنامه كاهش استرس مبتنى بر ذهن آَاهى به شيوه گروهى بر كيفيت زندكى ...

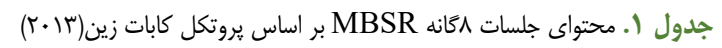

\begin{tabular}{|c|c|}
\hline اهداف و محتواى جلسات & جلسه \\
\hline و احعاسى برنامه و آهداف آن، توجه به هدايت خودكار و شناسايى آن (افكار عادتى، كليشه هاى ذهنى، طرح واره ها و ييش فرض ها)، توجه آكاهانه به واكنش ها، افكار & اول \\
\hline و ترمح منابع و عوامل ايجاد كننده فشار روانى و اضطراب، بررسى منابع استرس زاى اعضاى كروه، شرح واكنش هاى مناسب به فشارها و استرس هاى روزمره، آموزش & دوم \\
\hline 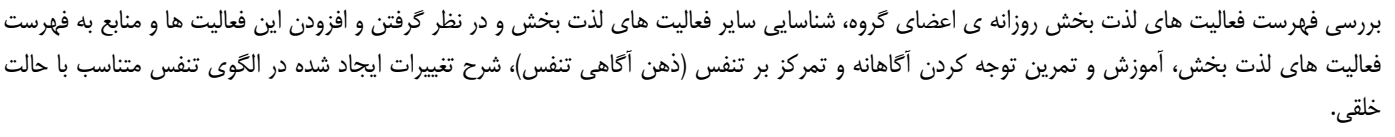 & سوم \\
\hline جوانب مثر زمان حال، هدايت افكار به بودن و ماندن در زمان حال، جلو خيرى از ورود افكار منفى، توجه آكاهانه به اطراف به عنوان يك تماشاكر و توجه به نكات و & جهارم \\
\hline 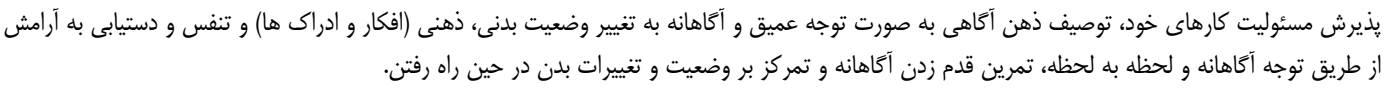 & ينجم \\
\hline 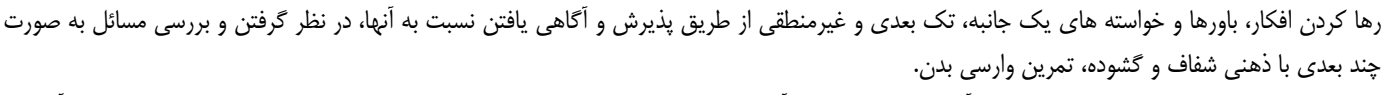 & 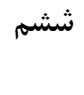 \\
\hline 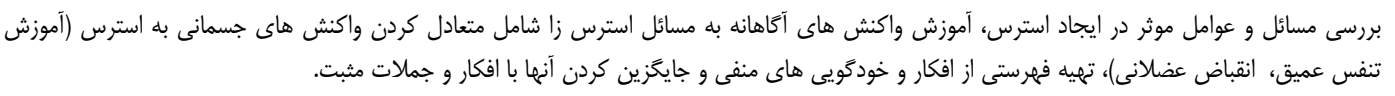 & هفتم \\
\hline 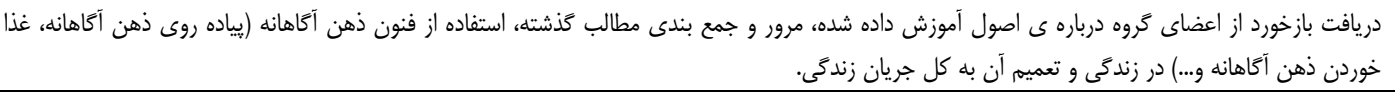 & هشتم \\
\hline
\end{tabular}

است. برايبررسى روايى سازه از تحليل عاملى اكتشافى إنى

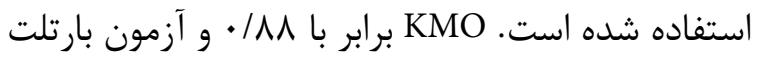

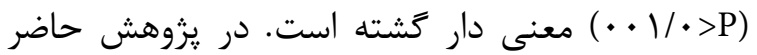

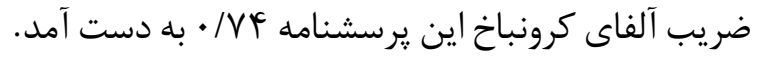

$$
\text { د. موش مداخله و شيوه اجرا }
$$

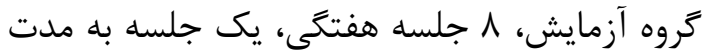

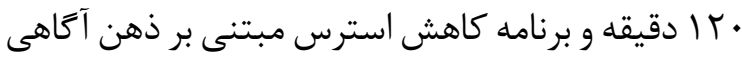

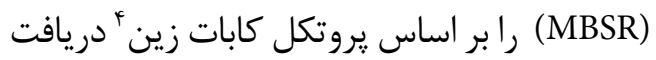

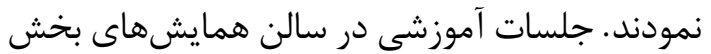

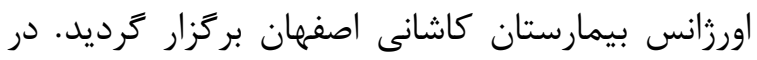

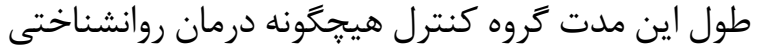

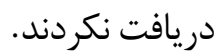

يافتله هاى يُزوهش در جدول شماره با آمار توصيفى مربوط بـ به ميانكين و

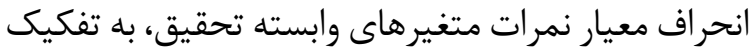

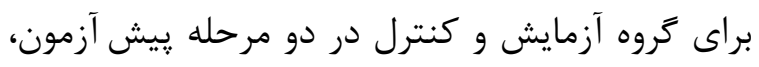
يس آزمون نشان داده شده است.

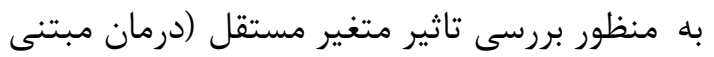

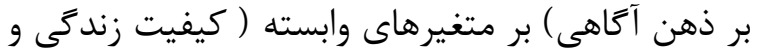

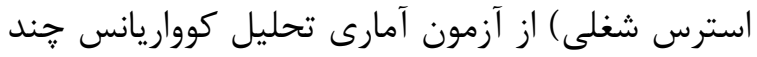

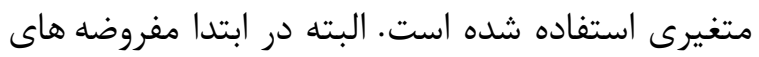
اين آزمون مورد بررسى قرار كرفت.

4 Kabat - Zinn
ه، و V داراى مقياس دو درجهاى البله و r خير هستند.

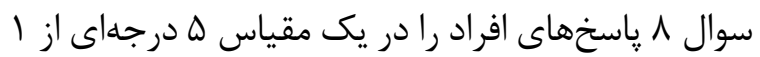

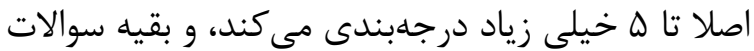

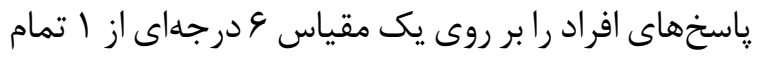

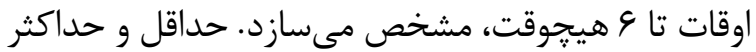

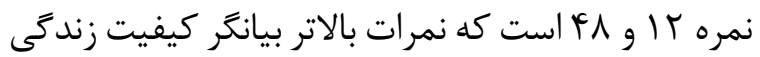

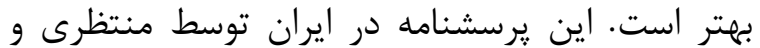

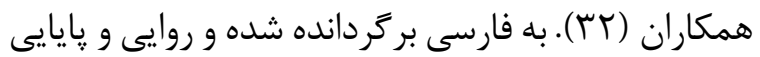

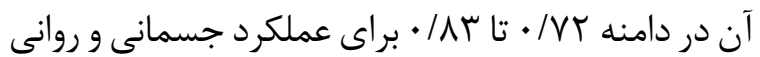

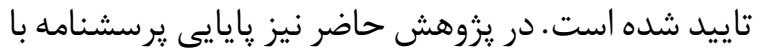

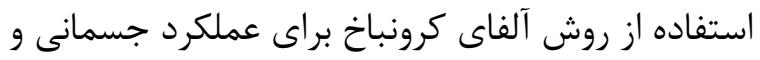

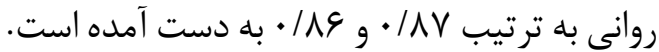

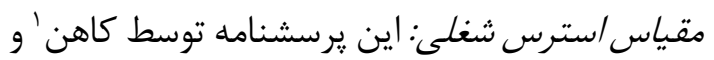

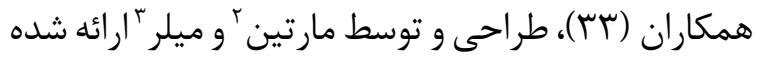

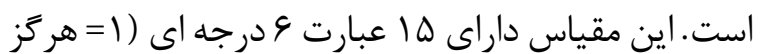

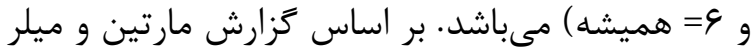

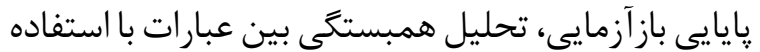

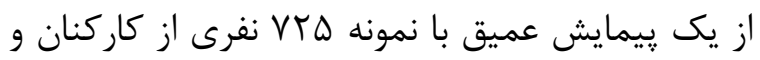

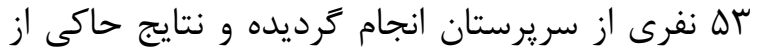

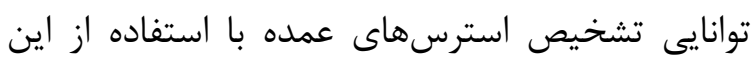

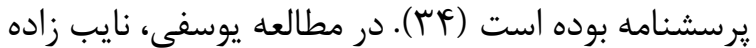

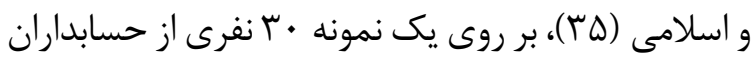

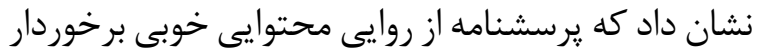


جدول r. ميانكَين و انحراف معيار كيفيت زندگى و استرس شغلى در دو كروه آزمايش و كنترل

\begin{tabular}{|c|c|c|c|c|c|c|c|c|}
\hline \multicolumn{4}{|c|}{ كروه كنترل } & \multicolumn{4}{|c|}{ كَروه آزمايش } & \multirow{3}{*}{ متغير } \\
\hline \multicolumn{2}{|c|}{ بֶس آزمون } & \multicolumn{2}{|c|}{ ييش آزمون } & \multicolumn{2}{|c|}{ يس آزمون } & \multicolumn{2}{|c|}{ ييش آزمون } & \\
\hline انحراف معيار & ميانگين & انحراف معيار & ميانگين & انحراف معيار & ميانگين & انحر اف معيار & ميانكين & \\
\hline $9 / 9$. & $V \varepsilon / \cdot \Delta$ & $r / \cdot 1$ & $V E / \cdot r$ & $q / 1 r$ & $99 / 1$. & $r / \mu$. & $V \pi / 4$. & كيفيت زندَّى \\
\hline $1 . / \pi$. & Fo/q & $V / r$. & $\lceil N / \Delta D$ & r & $T V / \Delta$. & g/Kr & kg/vg & استرس شغلى \\
\hline
\end{tabular}

جدول س. نتايج تحليل كوواريانس تاثير آموزش استرس مبتنى بر ذهن آَاهى بر ميزان نمرات كيفيت زندگى تكنسين هاى فوريت هاى بزشكى

\begin{tabular}{|c|c|c|c|c|c|c|}
\hline ميزان تاثير & معنادارى & $\mathbf{F}$ & ميانكين مجزورات & درجه آزادى & مجموع مجزورات & متغيرها \\
\hline.$/ .99$ & $\cdot / V T \Lambda$ & א & $\Delta r r / \cdot V$ & 1 & $\Delta r r / \cdot V$ & ييش آزمون \\
\hline $.199 \mathrm{~V}$ &.$/ .1$ & $\Delta \omega / v$. & rAVN/.1 & 1 & rNVN/. & متغير مستقل (آموزش) \\
\hline _- & - & - & $|A V V| \cdot \Lambda$ & Tr & $\mid \wedge V \gamma / \cdot \Lambda$ & خطا \\
\hline
\end{tabular}

جدولع. نتايج تحليل كوواريانس تاثير آموزش استرس مبتى بر ذهن آكاهى بر ميزان نمرات استرس شغلى تكنسين هاى فوريت هاى يزشكى

\begin{tabular}{|c|c|c|c|c|c|c|}
\hline ميزان تاثير & معنادارى & $\mathbf{F}$ & ميانكين مجزورات & درجه آزادى & مجموع مجزورات & متغير ها \\
\hline .1 .9 & $\cdot 11 \cdot 9$ & $r / 9$. & TrT/.r & 1 & TrT/.r & يِش آزمون \\
\hline$\cdot / \leftarrow \wedge \Delta$ & 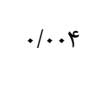 & $\mid r / v$. & אT/KITI & 1 & 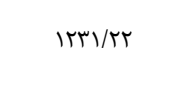 & متغير مستقل (آموزش) \\
\hline - & - & - & $\Delta r q / \Gamma q$ & Tr & $r r v .1 .0$ & خطا \\
\hline
\end{tabular}

كنترل) در مرحله يس آزمون تفاوت معنادارى وجود دارد.

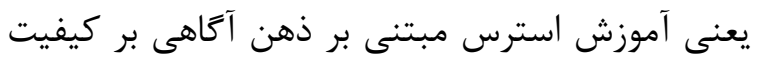

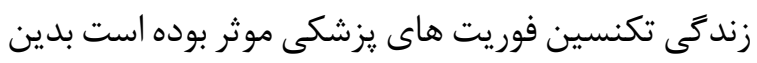

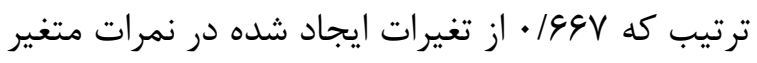

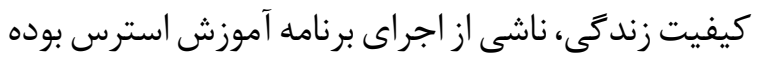
است. بر اساس يافتههاى جدول شماره أ، يس از حذف تاثير

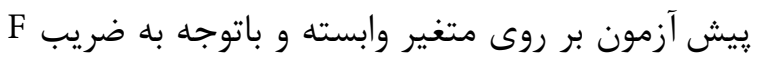

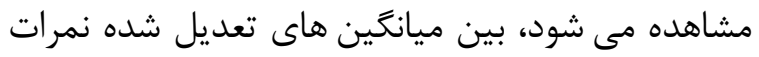

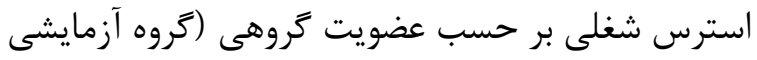

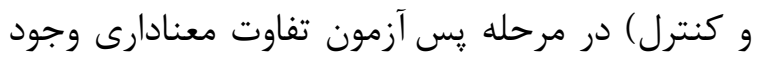
دارد. يعنى برنامه كاهش استرس مبتنى بر ذهن ذهن آناهى،

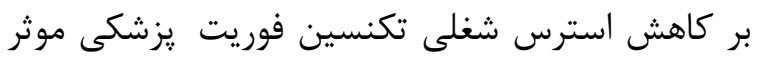

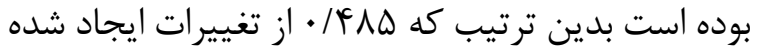
در نمرات متغير استرس شغلى، ناشى از اجراى مدئ مداخله بوده است.

\section{بحث و نتيجه تيرى}

يثوهش حاضر باهدف بررسى اثربخشى آموزش كاهش

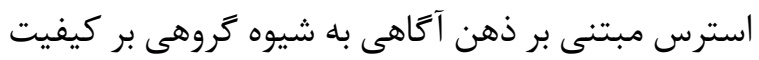

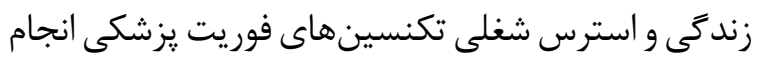

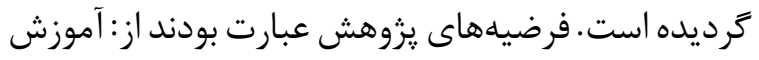

براى بررسى معنادارى تفاوت هاى مشاهده شده

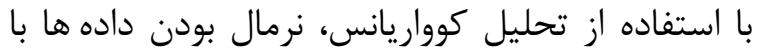

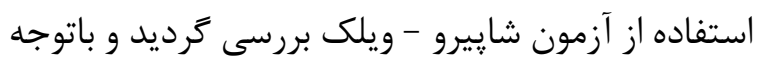
به اين كه سطح معنادارى براى متغيرهاى استير استرس

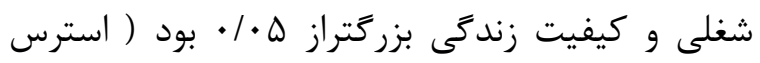

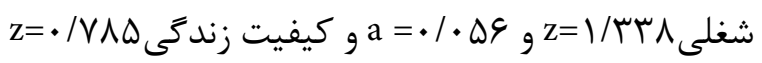

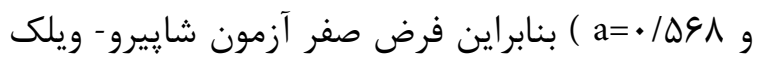

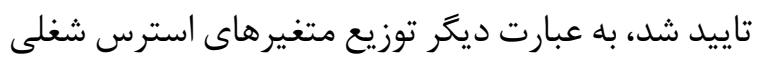

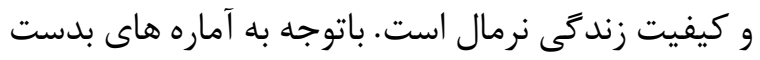

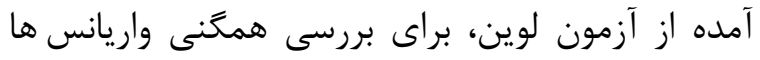

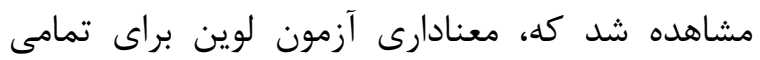

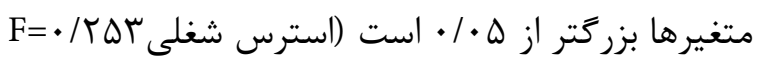
و لو a لذا فرض برابرى واريانس ها رعايت شده است. همجرنين

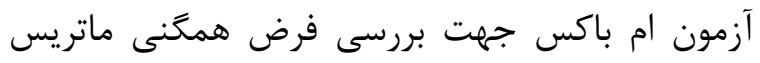
هاى واريانس - كوواريانس نشان داد كون كه اين مفروضه نيز برقرار است (a) مى توان از تحليل كوواريانس استفاده كرد.

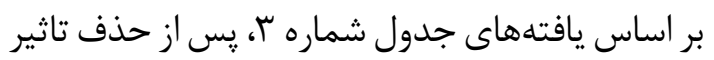

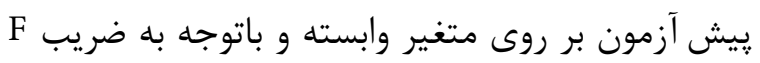

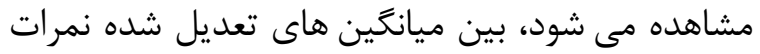
كيفيت زندگى بر حسب عضويت گروهى (كروه آزمايشى و 
اثربخشى برنامه كاهش استرس مبتنى بر ذهن آكاهى به شيوه كروهى بر كيفيت زندَى ...

حال، فنون كنترل بدن و ذهن و نغرشهاى كليدى غير

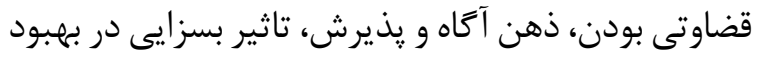

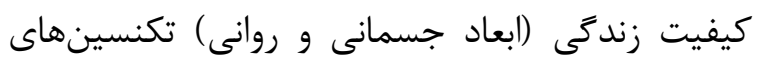

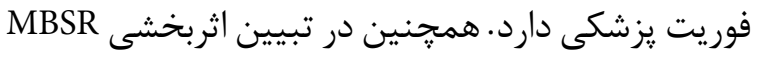

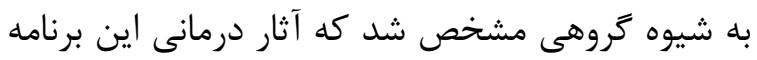

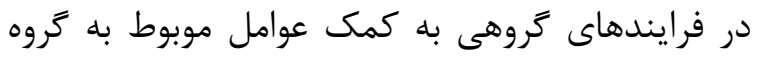

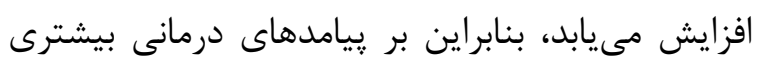

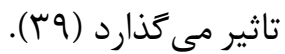

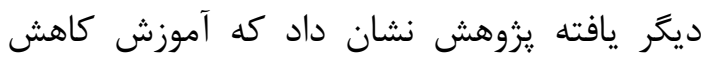

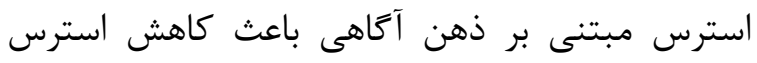

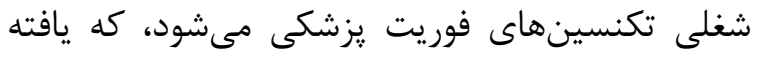

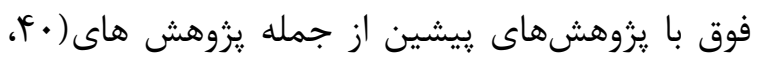

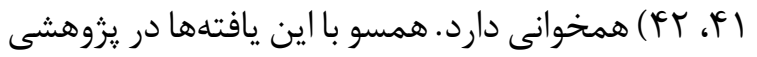

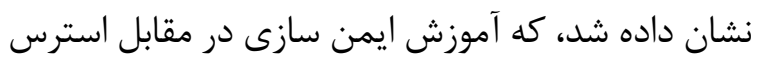

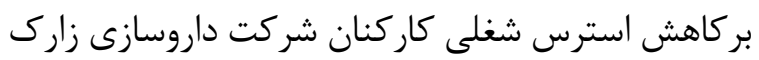

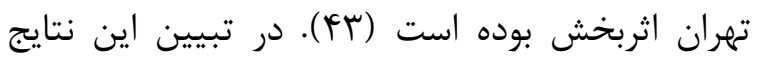

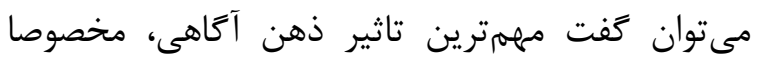

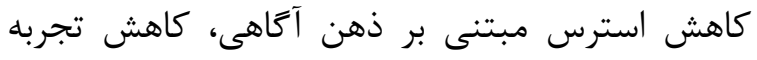

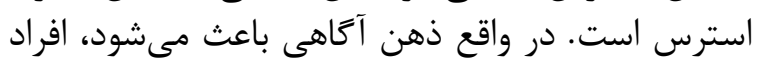

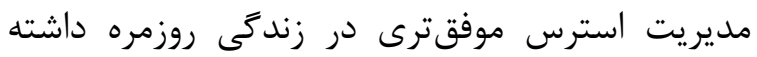

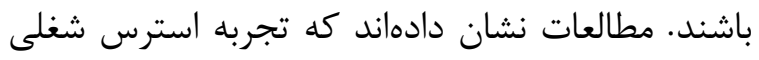

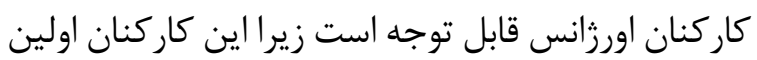

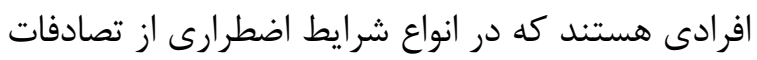

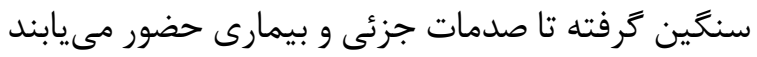

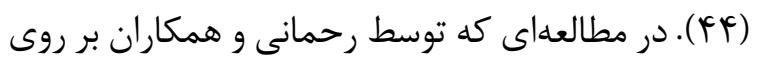

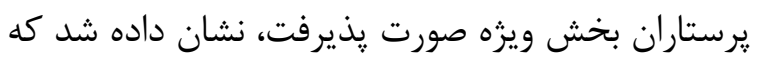

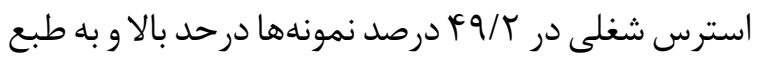

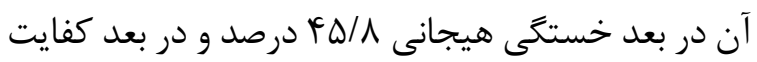

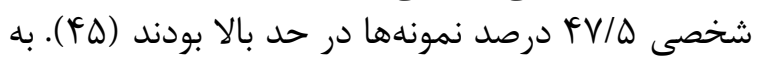

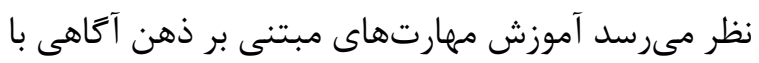

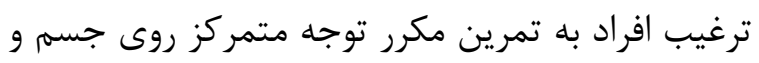

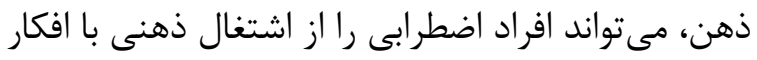

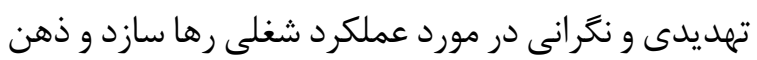

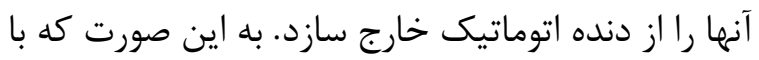

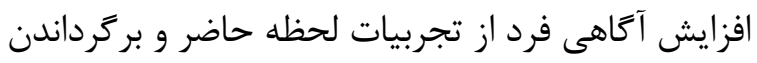

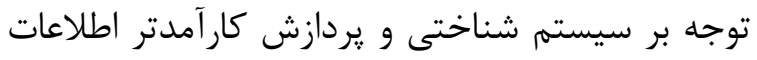

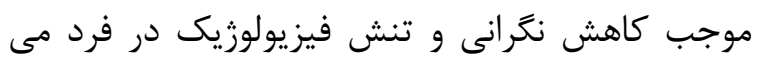

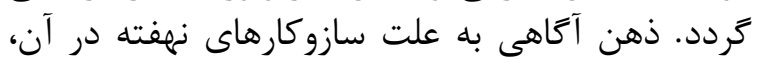

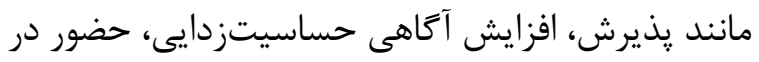

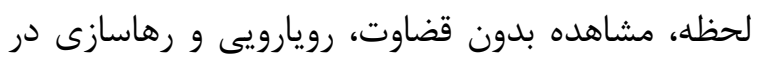

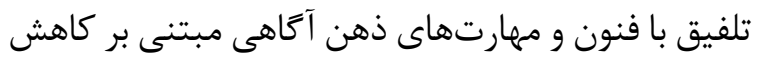

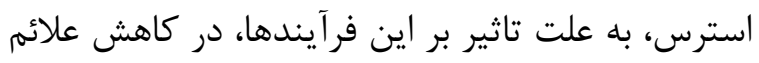

كاهش استرس مبتنى بر ذهن آكاهى در افزايش كيفيت

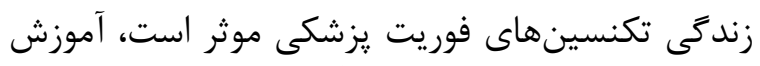

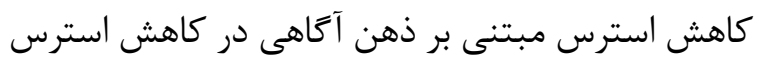

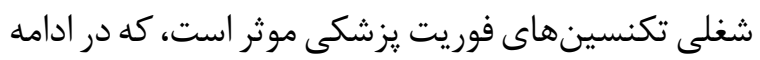

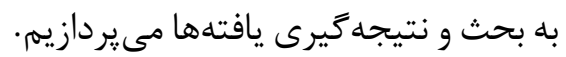

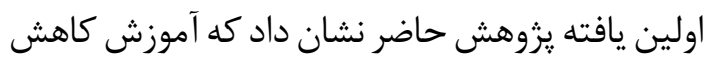

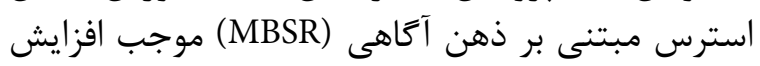

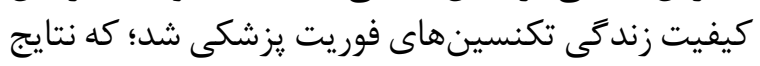

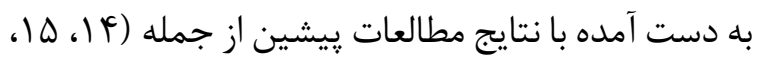

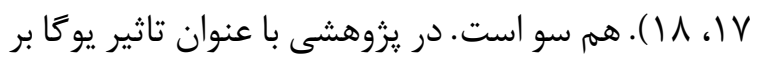

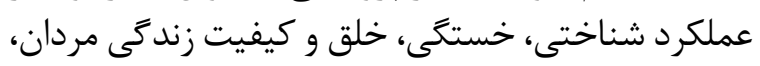

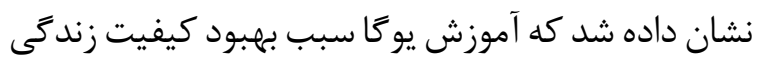

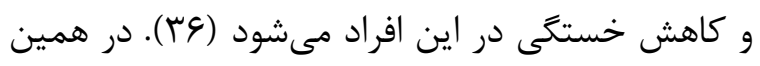

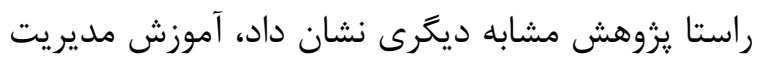

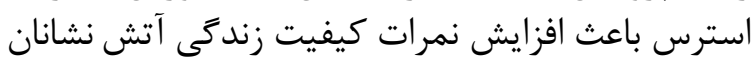

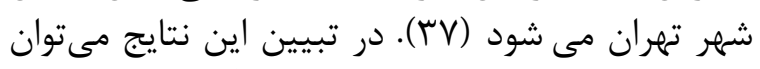

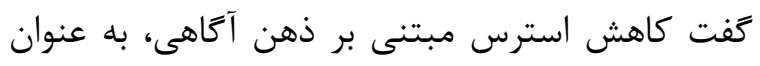

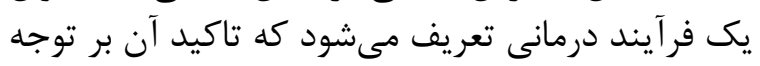

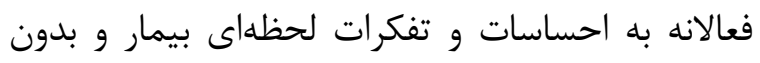

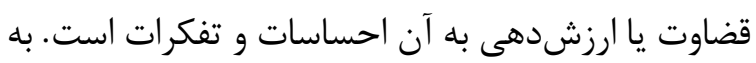

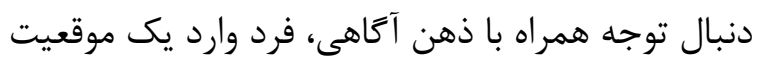

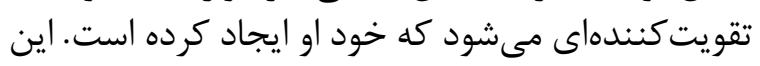

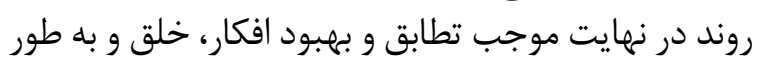

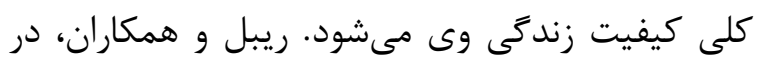

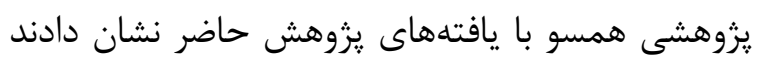

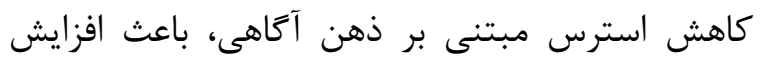

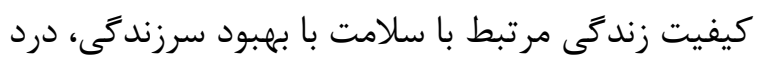

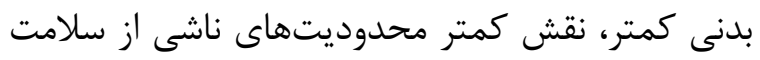

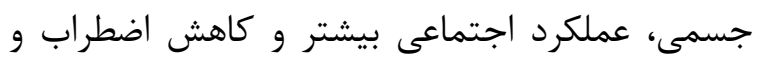

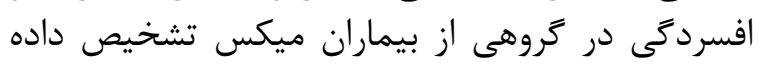

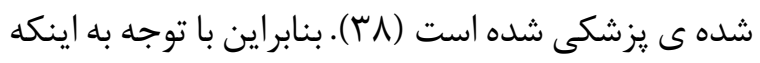

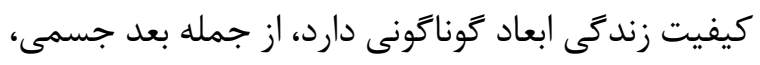

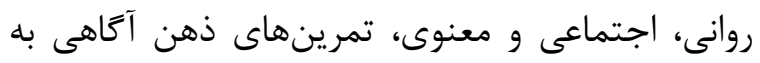

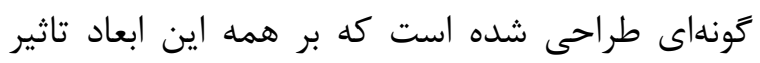

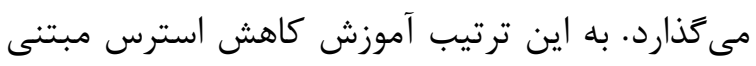

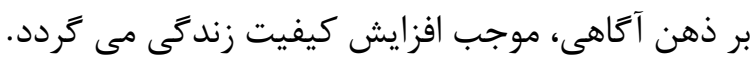

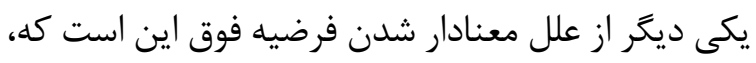

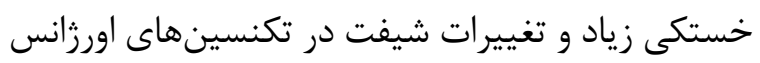

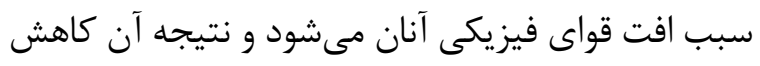

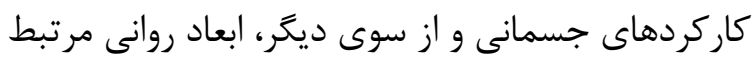

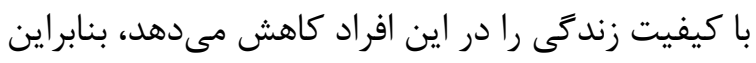

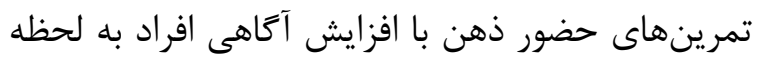


زندگى و تابآورى آنها در شرايط اورزانسى ارتقا يابد.

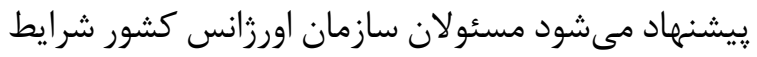

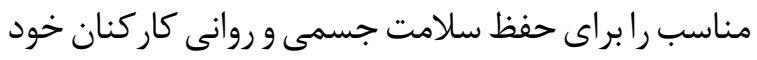

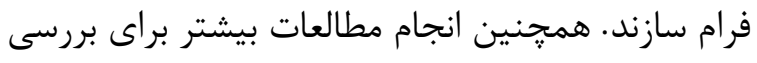

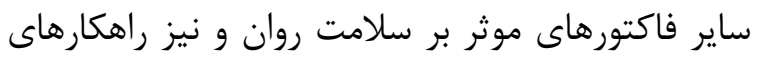

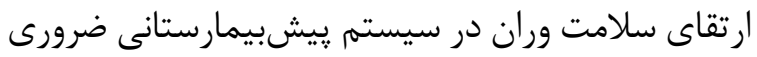
به نظر مىرسد.

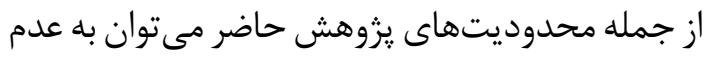

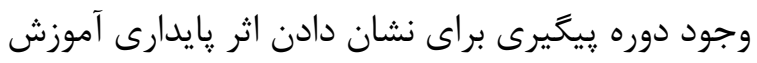

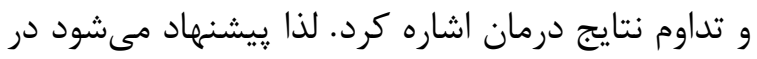

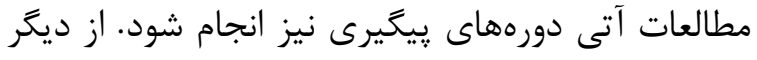

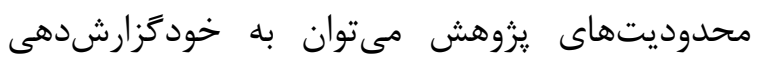

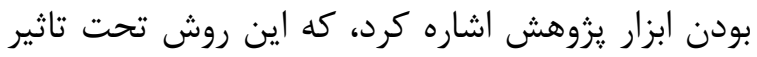

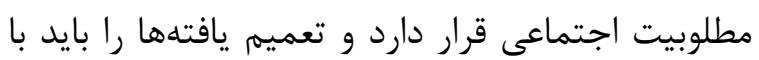

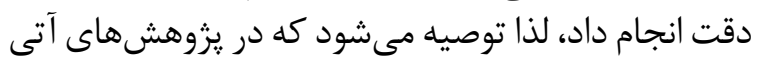

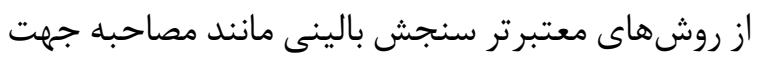
سنجش متغيرها و همجنين تقويت نقيت نتايج استفاده كردد.

تقدير و تشكر

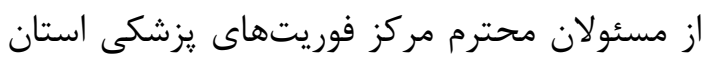

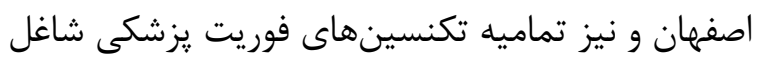

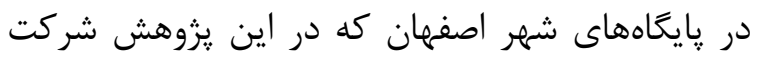

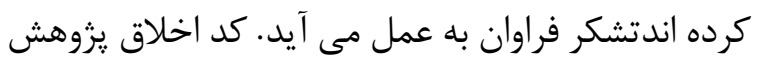

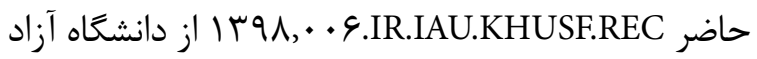
اسلامى واحد اصفهان (خوراسعان) است.

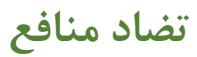

بدين وسيله نويسند

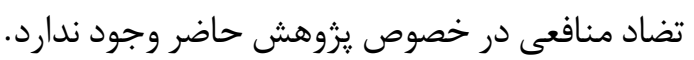

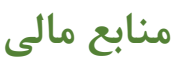

هيجَّونه كمك مالى براى انجام اين بزوهش دريافت

نشده است.

\section{References}

1. Rahmani F, Behshid M, Zamanzadeh V, Rahmani F. Relationships between general health, occupational stress and burnout in critical care nurses of Tabriz teaching hospitals. Iran J Nurs, 2010; 23(66):54-63. [persian]

2. Edwards FJ. Good Interdepartmental Relationships: The Foundations of a Solid Emergency Department. Advavced. Journal of Emergency Medicine, 2018; 2(2):e14.
بالينى استرس موثر بوده و از طرفى اثربخشى درمان رمان رادر

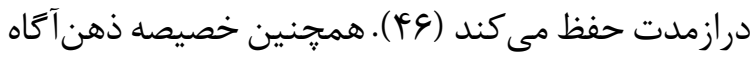

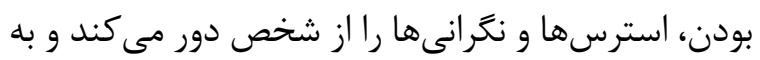

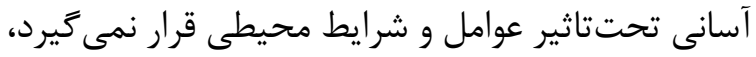

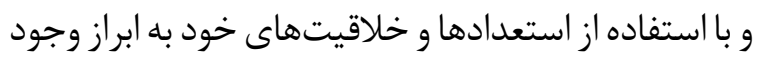

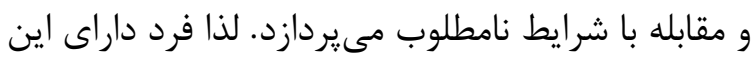

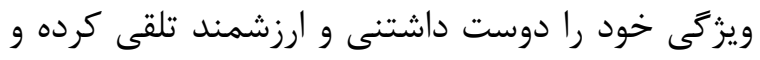

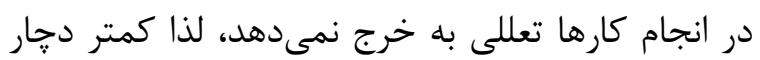

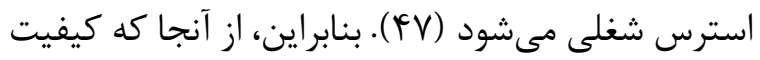

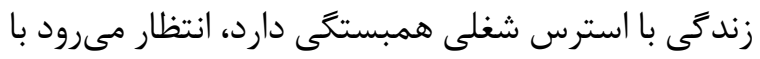

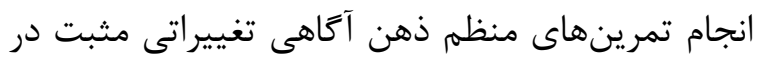

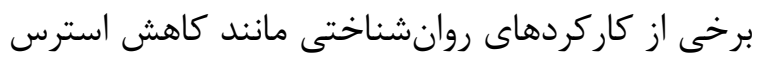

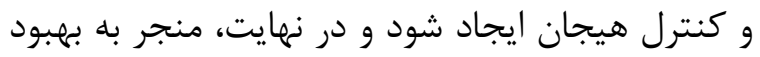

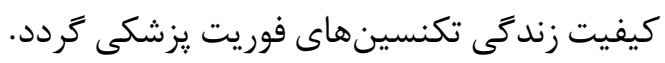

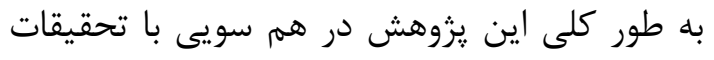

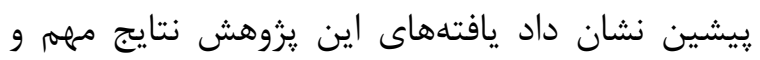

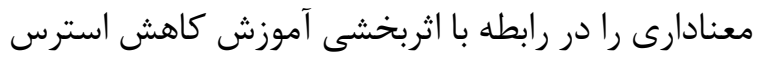

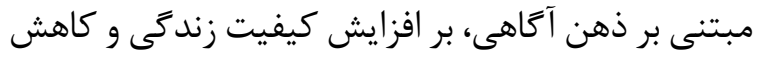

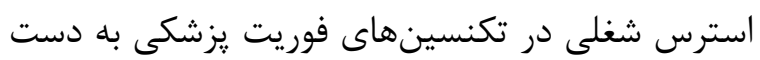

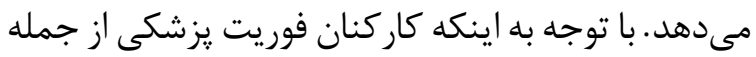

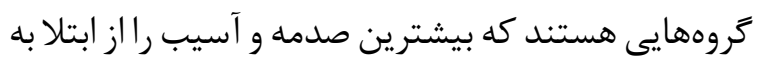

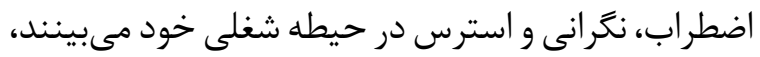

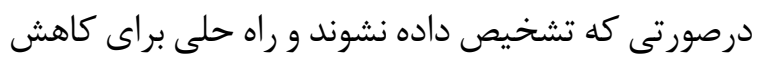

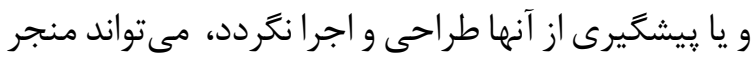

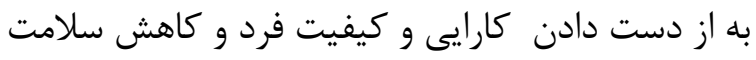

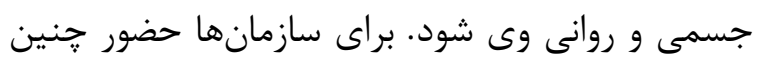

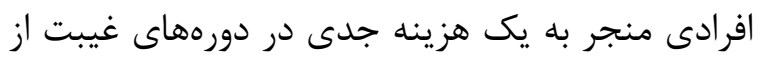

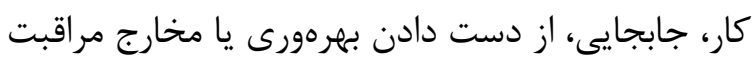

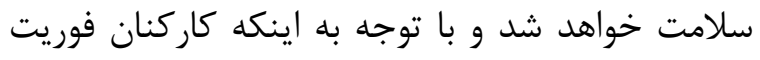

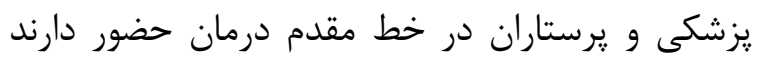

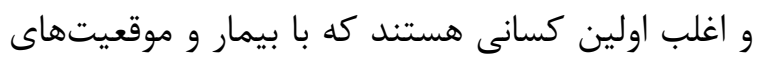

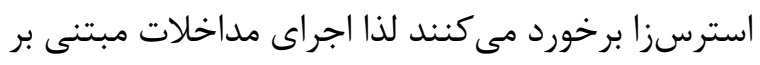

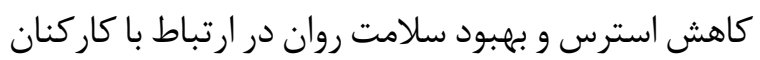

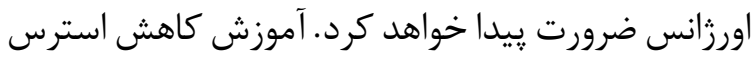

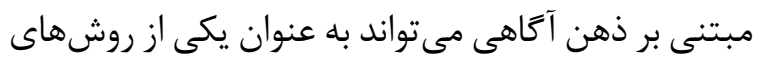

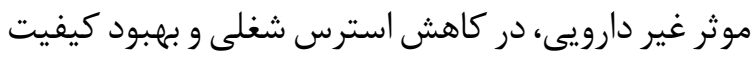

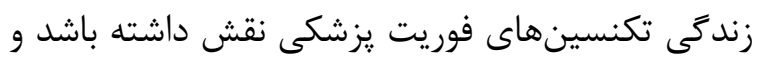

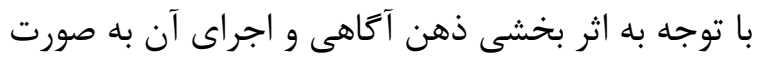

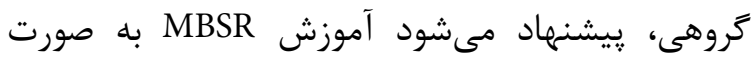

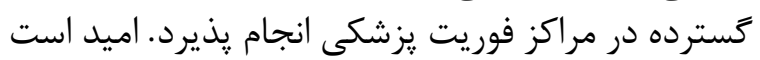

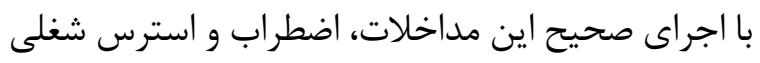
كاركنان اورزانس كاهش إين يابد و رضايت شاضي شغلى، كيفيت 
اثربخشى برنامه كاهش استرس مبتنى بر ذهن آكاهى به شيوه كروهى بر كيفيت زندگى ...

Life in Nurses. IJPN. 2017; 4 (5):1-7. [Persian]

16. Kabat-Zin J. There you are: Mindfulness meditation in everyday life. Wherever you go. New York: Hypersion; 1990.

17. Kabat-Zinn J. Full Catastrophe Living: Using the Wisdom of Your Body and Mind to Face Stress, Pain, and Illness. New York: Delacourt; 1990.

18. Kabat-Zinn J. An outpatient program in behavioral medicine for chronic pain patients based on the practice of mindfulness meditation. Theoretical considerations and preliminary results, Gen HospPsych, 1982; 4:33- 47.

19. Bishop SR. What do we really know about mindfulnessbased stress reduction? Psychosom Med 2002; 64: 7183.

20. Teasdale JD, Moore RG, Hayhurst H, Pope M, Williams $\mathrm{S}$, Segal ZV. Metacognitive awareness and prevention of relapse in depression: empirical evidence. J Consult Clinical Psycholgy, 2002; 70: 275-287.

21. Miller J, Fletcher K, Kabat-Zinn, J. Three-year follow-up and clinical implications of a mindfulness meditationbased stress reduction intervention in the treatment of anxiety disorders. Gen Hosp Psychiatry, 1995; 17(3): 192-200.

22. Heshmati R, Ghorbani F. The effect of MindfulnessBased Stress Reduction (MBSR) program on physical functioning and health related quality of life (HRQOL) in people with coronary artery disease (CAD), Cardiovascular Nursing Journal, 2016; 5(3): 16-25. [Persian]

23. Zarnaghash M, Mehrabizadeh H, Bashlide M, Davoodi $\mathrm{K}$, Marashi, Ali. The effect of mindfulness-based stress reduction therapy on quality of life and productivity of spouses of amputated veterans. Journal of Psychological Methods and Models, 2016; 7 (23): 83-99. [Persian]

24. Kabat-Zinn, J. Full Catastrophe Living: Using the Wisdom of Your Body and Mind to Face Stress, Pain and Illness (Revised and Updated Ed.). New York: The Random House Publishing Group: 2013; 720.

25. Wang W, Kong AWM, Chair SY. Relationship between job stress level \& coping strategies used by HONG KONG nurses working in an acute surgical unit. Applied Nursing Research, 2011; 24(4):238-43.

26. Augusto Landa J, López-Zafra E, Berrios Martos MP, ilar-Luzón Mdel C.The relationship between emotional intelligence, occupational stress and health in nurses. International Journal of Nursing studies, 2008; 45(6):888-901.

27. Abedi, K, Zare, M, Barkhordari, A, Halvani, GH. Work stress and job dissatisfaction and its relation to individual factors and accidents. Journal of Jahrom University of Medical Scinces, 2009; 7(2): 10-20. [persian]

28. Cosio D, Jinl, siddique J, Mohr DC. The effects of
3. Smith - Coggins R, Broderick KB, Marco CA, Night Shifts in emergency medicine: the american board of emergency medicine longitudinal study of emergency physicians. The Journal of emergency medicine, 2014; 47(3):372-8.

4. Mirbaha S, Forouzanfar M, Mohebi M, Kariman H. The Cost of Leaving the Emergency Department without Notice; a Cross-sectional Study, Advanced Journal of Emergency Medicine, 2018; 2(1):e6. [persian]

5. Ghaddar A, Mateo I, Sanchez p. occupational stress and mental health among correctional officers, J occupant health, 2008; 50(1):92-8.

6. Sofianopoulos S, Williams B, Archer F, Thompson B. The exploration of physical fatigue, sleep and depression in paramedics: a pilot study. Journal of Emergency primary Health care, 2011; 9(1):735.

7. Brough P. Comparing the influence of traumatic and organizational stressors on the psychological health of police, fire, and ambulance officers. International Journal of Stress Management, 2004; 11(3): 227-244.

8. Abbasi Esfjir AA, Amiri Mousavi S. Relationship between job stress and quality of life and marital adjustment among female doctors in Amol city. Journal of Women and Society, 2018; 9(3): 271-288. [persian]

9. Lee Y.H. Physical Discomfort and Psychological job stress Among Male and Female operators at Telemmunication call centers in Taiwan. European sociological Review, 2010; 40(4):561-8.

10. Ghazagh M, Zadhasan Z. The Effect of Group Mindfulness-Based Stress Reduction Program on the Quality of Life and Fatigue in Patients with Multiple Sclerosis. Avicenna J Nurs Midwifery care. 2019; 27 (1):35-44. [Persian]

11. Khosravi M. Examine job stressors librarians working in academic libraries Ministry of Science, Research and Technology, Tehran, from their perspective, research projects, Information and Documentation Center of Iran, Tehran, 2004.[Persian]

12. Brown, K, Ryan, R, Creswell, J. Mindfulness: theoretical Foundation and evidence for its salutary effect. Psychological Inquiry, 2017; 18(3):211-237.

13. Saberinia A, Aflatoniyan E, Jannat F, Aminizadeh M. The Effect of Mindfulness-Based Cognitive Therapy on Anxiety of Pre-Hospital Emergency Staff. jrehab. 2019; 20 (4):340-349. [Persian]

14. Jenaabadi H, Pilechi L, Salmabadi M, Tayarani Rad A. The Effectiveness of Training Mindfulness Skills in Professional Stress and Psychological Well-being of Female Teachers. ioh. 2017; 13 (6):58-69. [Persian]

15. mir rajaee $A$, mashhadi $A$, sepehri shamloo $Z$, shahidi sales S. The Effectiveness of Mindfulness-Based Stress Reduction on Perceived Stress, Resilience and Quality of 
Psychoneuroendocrinology. 2004; 29(4):448-74.

39. Speca M, Carlson LE, Goodey E, Angen M. A randomized, wait-list controlled clinical trial: the effect of a mindfulness meditation-based stress reduction program on mood and symptoms of stress in cancer outpatients. Psychosomatic medicine. 2000; 62(5):613-622.

40. Khadivi M, Zargar Y, Davoodi I. The effectiveness of cognitive-behavioral stress management training on type a behavior pattern and professional stress in workers of an industrial company. Journal of psychological achievements. 2012; 4(1):175-98. [Persian]

41. Hosseinaei A, Ahadi H, Fata L, Heidarei A, Mazaheri MM. Effects of Group Acceptance and Commitment Therapy (ACT)-Based Training on Job Stress and Burnout Iranian Journal of Psychiatry and Clinical Psychology. 2013; 19(2):109-20. [persian]

42. Hosseini z, Aghamollai T, Moeini B, Hazavehei SMM, Moghimbeigi A. The effect of health education program on femail nursses stress. Journal Health Syst Res. 2015; 11(1):641-6. [persian]

43. Makipour S, Shafiabadi A, Soudani M. The effectiveness of stress inoculation group training (SIT) on reducing job stress of employees of RAZAK pharmaceutical company in Tehran. Iran Occupational Health Journal. 2011; 7 (4):9-0. [Persian]

44. Essex B, Scott LB.Chronic stress and associated coping strategies among volunteers EMS personnel.prehospital Emergency care. 2008; 12(1):69-75.

45. Rahmani F, Behshid M, Zamanzadeh V, Rahmani F. Relationships between general health, occupational stress and burnout in critical care nurses of Tabriz teaching hospitals. Iran J Nurs. (2010);23(66):56-62 (Persian)

46. Jalali D, Aghaei A, Talebi H, Mazaheri MA. Comparison of the effectiveness of mindfulnessbased cognitive training appropriate with Iranian culture and the effectiveness of the cognitivebehavioral training on professional stress in employees of service firms. Journal of Occupational and Organizational Consulting. 2014; 6(18):114-38. [Persian]

47. Brown, A. (2015). Learning to learn: on tranining students to learn from text. Edu Res, 10(1), 14-24. telephone-administered cognitive- behavioral therapy on quality of life among patients with multiple sclerosis. Behavior Medical, 2011; 41(2). 227-34.

29. Gelamb G. K. The effectiveness of mindfulness on quality of life. Behavioral and cognitive Psychotherapy, 2011; 33(1), 103- 109.

30. Winzelberg A, Frederic M, Luskin MS. The effect of a meditation training in stress levels in school teachers. StressMedicine, 2012; 15(2): 69-77.

31. Rasoli M, Asodeh M H, Tamarchi F, Hosseini Z. Factor structure and validation of Self-Differentiation Inventory Short Form (SDI-SF). J Psychometry 2017; 4(17): 1-10. [Persian]

32. Montazeri A, Vahdaninia M, Mousavi SJ, Omidvari S. The Iranian version of 12- item Short Form Health Survey (SF-12): factor structure, internal consistency and construct validity. BMC public health 2009; 9(1): 341. [Persian]

33. Kahn RL, Wolf MD, Quinn PR, Snoek S, \& Rosenthal RA, Organizational stress: studies in role conflict and ambiguity. New York: Willey; 1964.

34. Martin JK, Miller GA. Job Satisfaction and Absenteeism: Organizational, Individual, and Job- Related Correlates. Work Occup, 1986; 13(1):33-46.

35. Yousefi S, Nayebzadeh S, Eslami H. The effects of job stress on Accountants job satisfaction. Iran Occupational Health, 2015; 12 (3):46-53. [Persian]

36. Oken BS, Zajdel D, Kishiyama S, Flegal K, Dehen C, Haas $M$, et al. Randomized, controlled, six-month trial of yoga in healthy seniors: effects on cognition and quality of life. Alternative therapies in health and medicine. 2006; 12(1):40.

37. Khodabakshi, AK, Falsafinejad, M R, Moghaddam, F, Mojarab, M. Stress management training on lifethreatening stressors and symptoms. Journal of Torbat Heydariyeh University of Medical Sciences, 2018; 6(1): 12-18. [persian]

38. Carlson LE, Speca M, Patel KD, Goodey E. Mindfulnessbased stress reduction in relation to quality of life, mood, symptoms of stress and levels of cortisol, dehydroepiandrosterone sulfate (DHEAS) and melatonin in breast and prostate cancer outpatients. 